\title{
Spectral narrowing via quantum coherence
}

\author{
Eugeniy E. Mikhailov, ${ }^{1}$ Vladimir A. Sautenkov, ${ }^{1,2}$ Yuri V. Rostovtsev, ${ }^{1}$ Aihua Zhang, ${ }^{1}$ M. Suhail Zubairy, ${ }^{1,3}$ \\ Marlan O. Scully, ${ }^{1,4}$ and George R. Welch ${ }^{1}$ \\ ${ }^{1}$ Department of Physics and Institute of Quantum Studies, Texas A\&M University, College Station, Texas 77843-4242, USA \\ ${ }^{2}$ P. N. Lebedev Institute of Physics, 119991 Moscow, Russia \\ ${ }^{3}$ Department of Electronics, Quaid-i-Azam University, Islamabad, Pakistan \\ ${ }^{4}$ Department of Chemistry, Princeton University, Princeton, New Jersey 08544, USA
}

(Received 29 November 2005; published 20 July 2006)

\begin{abstract}
We have studied the transmission through an optically thick ${ }^{87} \mathrm{Rb}$ vapor that is illuminated by monochromatic and noise-broadened laser fields in $\Lambda$ configuration. The spectral width of the beat signal between the two fields after transmission through the atomic medium is more than 1000 times narrower than the spectral width of this signal before the medium.
\end{abstract}

DOI: 10.1103/PhysRevA.74.013807

PACS number(s): 42.50.Gy, 42.50.Ar, 03.67.Hk

The resonant interaction of a quasimonochromatic electromagnetic field with atomic media is very important because of its applications to spectroscopy, magnetometry $[1,2]$, nonlinear optics [3], and quantum information and computing [4,5]. The interaction of a phase-noise-broadened electromagnetic field with media possessing a resonant absorption or transmission has been studied both experimentally and theoretically. In particular, several experiments involving noisy laser fields transmitted through a cell containing alkali-metal atomic vapor were performed. For example, the study of the conversion of phase noise to amplitude noise in dense $\mathrm{Cs}$ [6] and $\mathrm{Rb}$ [7] confirmed the theoretical prediction in [8]. In addition, there are experimental studies of intensity fluctuations and correlations between the drive and probe fields in the electromagnetically induced transparency (EIT) regime [9-12]. So a coherently driven medium cannot be treated as a simple well-defined filter; moreover such treatment leads to the wrong conclusion that the EIT transparency window should be wider with noisy laser fields [13]. Thus interaction of noisy laser fields with the EIT medium must be taken into account with great care.

In this paper, we show that the spectral width of the beat signal between two lasers is modified by transmission through a medium to give a very narrow spectral feature that is more than 1000 times narrower than the spectral width of the probe laser radiation. This can find broad applications in spectroscopy to develop light sources that have a very narrow (less than natural) spectral line whose carrier frequency ranges from optical to gamma ray where experimental demonstration of EIT has been recently reported [14]. It is important to note here that the spectral width of the line and the coherence time of radiation is controlled by an auxiliary external laser field. Below we provide a treatment of this effect alternative to that of [13], which explains the experimental observation of the spectral width narrowing of the noisy input probe field. It is also worth mentioning that the current technique can be applied to a single-photon source (see, for example, [10]) and allows one to transfer the flux of single photons with a particular or not defined coherence time to the flux of single photons with a given coherence time.

Our experimental setup is shown in Fig. 1(a). The laser is tuned to the transition [(a) $F=2-$ (c) $F=2$; see Fig. 1(b)], and the its output is modulated by an electro-optic modulator
(EOM) which is driven at the frequency of the ground-level splitting [6.835 GHz; see Fig. 1(b)]. Two sidebands are generated, one with the frequency of the probe field and another with frequency downshifted by $6.835 \mathrm{GHz}$ with respect to the drive field. This downshifted field is far from resonance and has a negligible effect on the experiment. The power and frequency of the sidebands are varied by changing the frequency and amplitude of the microwave field driving the EOM. If the EOM is driven by a spectrally narrow microwave source, then we observe a narrow EIT spectrum, just as for the case when two phase-locked lasers are used [15].

After the EOM, optical fields are sent through a singlemode optical fiber to make a clean spatial intensity distribution with diameter $0.7 \mathrm{~cm}$. The optical fields are circularly polarized by a quarter-wave plate. Then the drive and phasenoise-broadened probe beams propagate through a cell containing atomic ${ }^{87} \mathrm{Rb}$ at temperature $67.7^{\circ} \mathrm{C}$ and a buffer gas, neon, at pressure 30 Torr; the length of the cell is $2.5 \mathrm{~cm}$; the diameter is $2.2 \mathrm{~cm}$. The power at the entrance of the cell is $0.58 \mathrm{~mW}$; after the cell power is $0.32 \mathrm{~mW}$. We use heterodyne detection of the probe by mixing the transmitted light with an additional field that is frequency shifted by an acousto-optic modulator at $60 \mathrm{MHz}$ with respect to the drive field. This field does not propagate through the ${ }^{87} \mathrm{Rb}$ medium. This detection technique has been described in [15]. A spectrum analyzer tuned in the vicinity of the beat-note frequency of the probe and additional fields is used to record the spectrum of the beat note, which coincides with the spectral density of the phase-noise-broadened transmitted probe field for the case of a monochromatic drive field.

In the current experiment, the EOM is modulated with a broad ("white") noise spectrum centered about the selected probe frequency. The total power in each sideband is about $10 \%$ of the power in the drive field, and the spectral width of the beat signal between the probe and drive is $\sim 1 \mathrm{MHz}$. We can characterize the modulation of the EOM as a timedependent frequency $f(t)=f_{0}+\Delta f(t)$ where $f_{0}$ is the carrier frequency $(6.835 \mathrm{GHz})$, and $\Delta f(t)$ is the noise-driven timedependent frequency shift. Since the phase of such an oscillation is equal to $\varphi(t)=\varphi_{0}+f_{0} t+\int_{-\infty}^{t} \Delta f(t) d t$, we have a phasenoise-broadened microwave signal that drives the EOM. Thus we have a phase-noise-broadened probe field instead of 

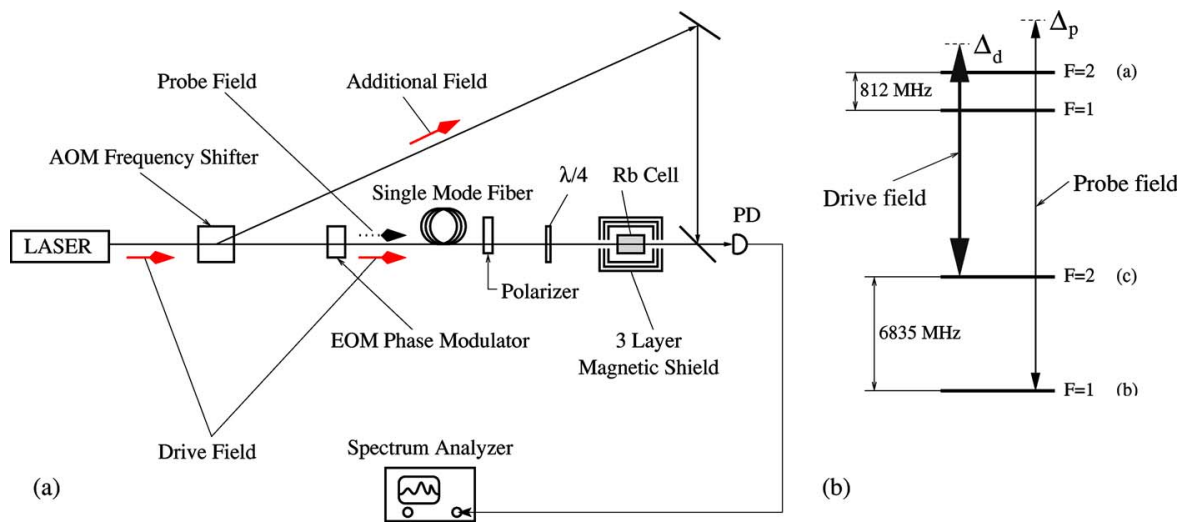

(b)

a monochromatic probe field which is observed without the phase noise modulation. This is equivalent to using two lasers, one of which is spectrally narrow, and the other of which has a phase-noise-broadened spectrum.

We note that the spectral density of the probe signal does not coincide exactly with the spectrum of the driving microwave signal because of the limited bandwidth of the EOM response to the modulation frequency. A typical plot of spectral density is depicted in Fig. 2. Before the cell, the spectral full width at half maximum (FWHM) of the probe field is $980 \mathrm{kHz}$, whereas after the cell we see significant narrowing of the spectrum with a FWHM of $4.6 \mathrm{kHz}$ (see also Fig. 3). Before the cell, we have a Gaussian distribution of the spectral density,

$$
f_{\text {in }}(\omega)=e^{-\left(\omega-\omega_{0}\right)^{2} / \omega_{w}^{2}}
$$

where $\omega_{0}$ is the average probe field frequency and $\omega_{w}$ is the width of the spectral density spectrum. However, after the cell, the probe field has a Lorentzian distribution

$$
f_{\text {out }}=\frac{\gamma_{n}^{2}}{\left(\omega-\omega_{0}\right)^{2}+\gamma_{n}^{2}}
$$

where $\gamma_{n}$ is the width of the transmitted spectral density spectrum.

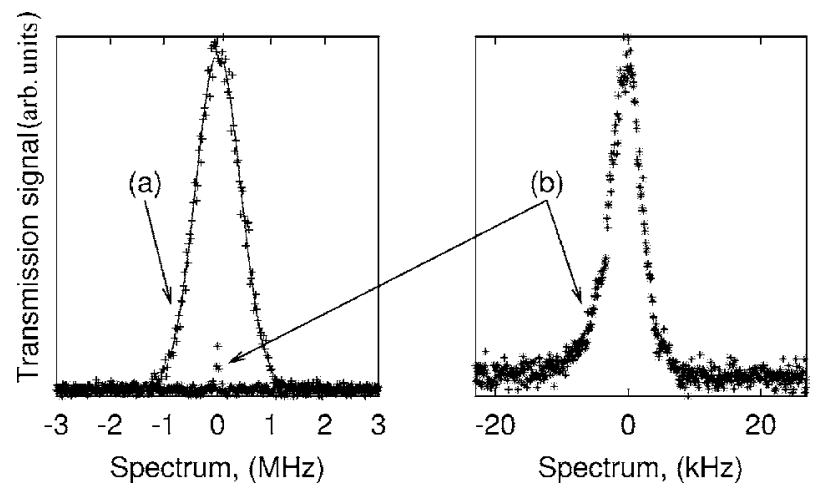

FIG. 2. Spectral density of the phase-noise-broadened probe laser after passing the ${ }^{87} \mathrm{Rb}$ cell. (a) shows the spectrum with the lasers far from resonance, so that the interaction with the atoms is negligible. (b) shows the spectrum with the lasers on resonance. The solid line in (a) is a Gaussian fit.
FIG. 1. (Color online) (a) Experimental setup. (b) Atomic level structure for ${ }^{87} \mathrm{Rb}$.
In Fig. 3 we compare transmission spectra taken under two experimental conditions: First, we measure the usual EIT resonance with coherent drive and probe fields by scanning the two-photon detuning between the two coherent fields. Second, we use the phase-noise-broadened probe and measure the spectral density of the transmitted light. Note that the measured spectra after normalization coincide within our experimental accuracy, which means that $\gamma_{n}=\gamma_{\text {EIT }}$ where $\gamma_{\text {EIT }}$ is the width of the EIT measured with the coherent probe and drive fields.

It is interesting to note that we significantly increase the correlation time of the output probe field $\tau_{\text {out }}$ with respect to the correlation time of the ingoing probe field $\tau_{\text {in }}$. The characteristic coherence time $\tau_{\text {in }}=2 / \omega_{w}$ for the input probe field is much smaller than the characteristic coherence time of the output radiation $\tau_{\text {out }}=2 / \gamma_{n}=2 / \gamma_{\text {EIT }}$, as $\omega_{w} \gg \gamma_{\text {EIT }}$. Thus, we can create a source of radiation with controllable coherence time. This point is illustrated in Fig. 4, where we measure the dependence of the transmitted spectral width of the signal as a function of the power of the drive laser.

In order to understand the surprising fact that the spectral density of the beating signal does not significantly depend upon the spectral widths of the drive and the probe fields, we first recall that the phase-noise-broadened probe and drive

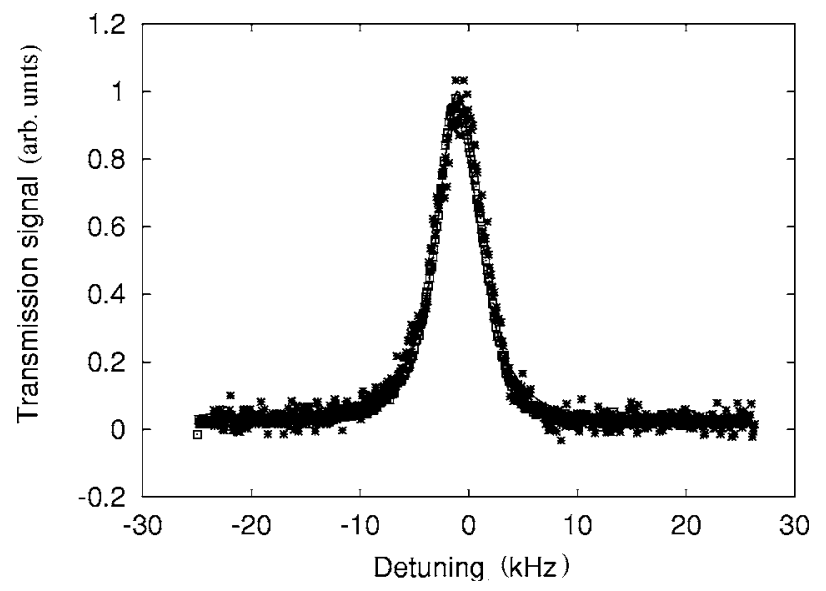

FIG. 3. Comparison of the EIT resonance obtained two ways. Data points marked with squares are transmission versus probe laser frequency for spectrally narrow drive and probe fields. Data points marked with stars are the spectrum of the transmitted probe light when the probe is phase broadened. 


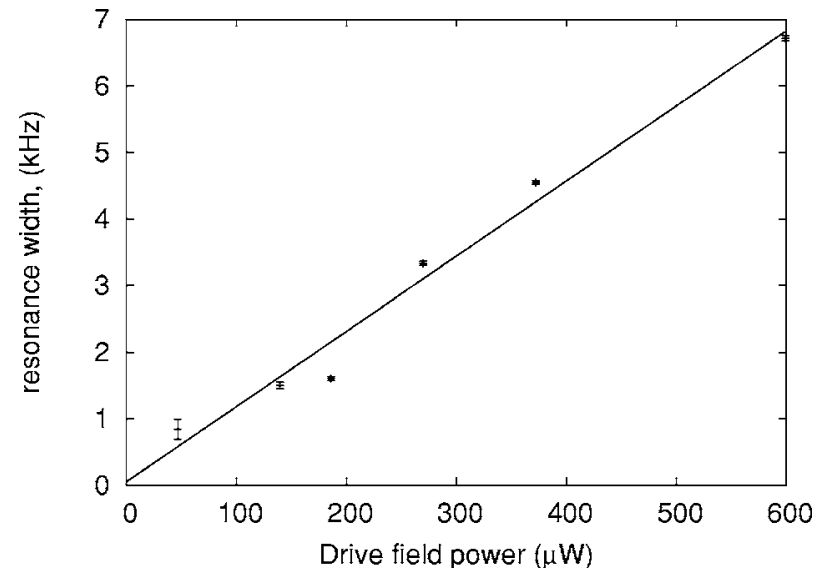

FIG. 4. Dependence of spectral width of the transmitted probe field (see Fig. 3) vs power of the drive laser under the condition of EIT resonance. Thus, the coherence time of the probe field can be controlled by the power of the drive field.

field can be written as $E_{p}(t)=E_{p 0} e^{-i\left(\omega_{p} t+\varphi_{p}(t)\right)}, E_{d}(t)$ $=E_{d 0} e^{-i\left(\omega_{d} t+\varphi_{d}(t)\right)}$. If the phase fluctuations are random, then $\left\langle\varphi_{d}\right\rangle=0, \quad\left\langle\varphi_{p}\right\rangle=0, \quad\left\langle\dot{\varphi}_{d}(t) \dot{\varphi}_{d}\left(t^{\prime}\right)\right\rangle=2 D_{d} \delta\left(t^{\prime}-t\right)$, and $\left\langle\dot{\varphi_{p}}(t) \dot{\varphi_{p}}\left(t^{\prime}\right)\right\rangle=2 D_{p} \delta\left(t^{\prime}-t\right)$. Thus, as was shown in [13], the EIT resonance is broadened by the quantity $D_{p}+D_{d}$.

Our experimental results can be explained by using a simplified three-level structure for ${ }^{87} \mathrm{Rb}$ atoms as depicted in Fig. 1. We let levels $a, b$, and $c$ correspond to ${ }^{87} \mathrm{Rb}$ atomic levels $5 P_{1 / 2} F=2,5 S_{1 / 2} F=1$, and $5 S_{1 / 2} F=2$, respectively. The driving field couples levels $a$ and $c$ and the probe field couples levels $a$ and $b$. The interaction picture Hamiltonian for the system can be written as

$$
\hat{H}=\hbar \Omega_{d} e^{i \Delta_{d} t}|a\rangle\left\langle c\left|+\hbar \Omega_{p} e^{i \Delta_{p} t}\right| a\right\rangle\langle b|+\text { H. c. }
$$

where $\Delta_{p}=\omega_{a b}-\nu_{p}$ and $\Delta_{d}=\omega_{a c}-\nu_{d}$ are the detunings of probe and drive fields from the atomic transitions $a-b$ and $a$ $c$, respectively, $\nu_{p}$ and $\nu_{d}$ are the frequencies of the probe and drive fields, $\Omega_{d}=\wp_{a c} E_{p} / \hbar$ and $\Omega_{p}=\wp_{a b} E_{p} / \hbar$ are the Rabi frequencies, and $\wp_{a b}$ and $\wp_{a c}$ are the dipole moments of the $a-b$ and $a-c$ transitions.

We are interested in finding the spectrum of the beat signal $S(t, z)=\Omega_{p}(t, z) \Omega_{d}^{*}(t, z)$ that is governed by the propagation equation along the $z$ axis $(0<z<L=2.5 \mathrm{~cm})$

$$
\frac{\partial S}{\partial z}=-i \eta \rho_{a b} \Omega_{d}^{*}+i \eta \rho_{c a} \Omega_{p}
$$

where $\rho_{a b}$ and $\rho_{c a}$ are the density matrix elements; $\eta$ $=3 \lambda^{2} N \gamma_{r} / 8 \pi ; N$ is the atomic density; $\gamma_{r}$ is the radiative decay from level $a$ to level $b ; \lambda=2 \pi c / \omega_{a b}$.

The adiabatic approximation can be used to find the coherences $\rho_{a b}$ and $\rho_{c a}$, because the bandwidth of the phasenoise is less than the relaxation rate of these coherences. Under these conditions, the propagation of the correlation function for the beat signal, $R(\tau, z)=\langle S(t, z) S(t+\tau, z)\rangle$, and the time dependence of the correlation of beat signal and spin coherence, $G(\tau, z)=\left\langle S(t, z) \rho_{c b}(t+\tau, z)\right\rangle$, are governed by

$$
\begin{aligned}
\frac{\partial}{\partial z} R(\tau, z)= & 2 \eta\left(\frac{n_{a b}}{\Gamma_{a b}}-\frac{n_{c a}}{\Gamma_{c a}}\right) R(\tau, z) \\
& -2 \eta\left(\frac{\left|\Omega_{d}\right|^{2}}{\Gamma_{a b}}+\frac{\left|\Omega_{p}\right|^{2}}{\Gamma_{c a}}\right) G(\tau, z), \\
\frac{\partial}{\partial \tau} G(\tau, z)= & \left(\frac{n_{a b}}{\Gamma_{a b}}-\frac{n_{c a}}{\Gamma_{c a}}\right) R(\tau, z) \\
& -\left(\Gamma_{c b}+\frac{\left|\Omega_{d}\right|^{2}}{\Gamma_{a b}}+\frac{\left|\Omega_{p}\right|^{2}}{\Gamma_{c a}}\right) G(\tau, z) .
\end{aligned}
$$

Here $\Gamma_{a b}=\gamma_{a b}+i \Delta_{p}$ and $\Gamma_{c a}=\gamma_{a c}-i \Delta_{a c}$ where $\gamma_{a b}$ and $\gamma_{a c}$ are the relaxation rates of atomic coherences $\rho_{a b}$ and $\rho_{a c}$, respectively. Also $n_{a b}=\rho_{a a}-\rho_{b b}$ and $n_{c a}=\rho_{c c}-\rho_{a a}$ are the population differences. We have assumed a slow variation of atomic populations such that they do not change appreciably during propagation. A detailed analysis justifying this approximation will be presented elsewhere. It is clear that under these approximations, the correlation function of the beat signal (and hence the corresponding spectral density) is independent of the phase fluctuations of the drive and probe fields.

Let us note the important role of atomic coherence in the discussed processes. Before we proceed to solving a set of coupled Eqs. (5) and (6), note that the atomic coherence plays an important role in this processes, once induced it gives rise to a term $G(\tau, z)$. In the adiabatic limit, one can obtain transparency behavior governed by

$$
\frac{\partial}{\partial z} R(\tau, z)=2 \eta \mathcal{N} \frac{\Gamma_{c b} R(\tau, z)}{\widetilde{\Gamma}_{c b}}
$$

if the condition $\left|\Omega_{d}\right|^{2} \gg \Gamma_{a b} \Gamma_{c b}$ is met. Here we introduce $\tilde{\Gamma}_{c b}=\Gamma_{c b}+\frac{\left|\Omega_{d}\right|^{2}}{\Gamma_{a b}}+\frac{\left|\Omega_{p}\right|^{2}}{\Gamma_{c a}}$ and $\mathcal{N}=\frac{n_{a b}}{\Gamma_{a b}}-\frac{n_{c a}}{\Gamma_{c a}}$.

In order to determine the spectral density of the beat signal $I_{\omega}$, we recall the definitions

$$
R(\tau, z)=\int I_{\omega}(z) e^{-i \omega \tau} d \omega, \quad G(\tau, z)=\int \rho_{\omega}(z) e^{-i \omega \tau} d \omega
$$

Substituting these expressions for $R(\tau, z)$ and $G(\tau, z)$ into Eqs. (5) and (6) we find

$$
\rho_{\omega}=\frac{\mathcal{N} I_{\omega}}{\widetilde{\Gamma}_{c b}-i \omega} \text { and } \frac{\partial}{\partial z} I_{\omega}=2 \eta \frac{\left(\Gamma_{c b}-i \omega\right) \mathcal{N}}{\tilde{\Gamma}_{c b}-i \omega} I_{\omega} .
$$

For the simplest case, with a weak probe and a strong drive, $\left|\Omega_{d}\right| \gg\left|\Omega_{p}\right|$ and all population remains in state $b$, so that $n_{b}=1, n_{a}=n_{c}=0$. Taking Doppler broadening into account by integration over the velocity distribution leads to changing the homogeneous width $\gamma\left(2 \times 10^{7} \mathrm{~s}^{-1}\right)$ to the Doppler width $\Delta_{W}(2 \pi \times 500 \mathrm{MHz})$. Also, there is narrowing because the medium is optically thick [16]. The spectral density of the beat signal is then given by 


$$
I_{\omega}(z)=I_{\omega}(0) \exp \left(\frac{-\eta z \omega^{2}}{\Delta_{W}\left[\left(\frac{|\Omega|^{2}}{\Delta_{W}}\right)^{2}+\omega^{2}\right]}\right),
$$

with the following expression for the spectral width of the beating signal:

$$
\Delta \omega_{b s}=\frac{|\Omega|^{2}}{\Delta_{W} \sqrt{\frac{\eta z}{\Delta_{W}}-1} .}
$$

This expression agrees very well with our experimental results, namely, it gives a linear dependence of the spectral width on the driving power, and the same slope for the experimental parameters and a density of atoms of the order of $3 \times 10^{11} \mathrm{~cm}^{-3}$, which corresponds to the cell temperature. Note that for the case of monochromatic drive and broadened probe field, the spectral width of the beating signal coincides with the spectral width of the EIT for a monochromatic probe field. Let us again stress here that the obtained results both experimental and theoretical are different from one would expect on the basis of the results obtained for noisy fields [13]. The results obtained here can be interpreted in the sense that the probe field passing the cell and the atomic coherence in the cell are strongly correlated [see Eq. (8)] [17]. It is also worth mentioning that an interesting aspect of the problem was studied recently in $[11,12]$, where it was demonstrated that, when probe and drive intensities are of the same order of magnitude, the noise of the probe and drive fields is strongly correlated [11], and the phase noise of one laser is transferred to the second laser [12]. Also we would like to underline that the results obtained here go beyond the classical EIT treatment [13] where the broadening of EIT resonance was predicted.

In conclusion, we have experimentally observed that the width of the beat signal between a coherent drive and broadband probe field is greatly reduced (more than 1000 times) by propagation through a cell containing ${ }^{87} \mathrm{Rb}$ vapor. We analyzed the modification of a broad emission spectral line after transmission through a coherently prepared resonant medium. The final spectral line shape is defined by the spectral shape of the EIT resonance. The applications of the results obtained might be light sources (including singlephoton-sources) [10] with controllable coherent time of radiation, and notch filters with a subnaturally narrow transmission band that are in great demand for many practical applications to background suppression in imaging including astrophysics and environmental imaging [18].

\section{ACKNOWLEDGMENTS}

We thank S. E. Harris, M. D. Eisaman, M. D. Lukin, P. Meystre, and A. S. Zibrov for useful discussions and gratefully acknowledge the support from the Office of Naval Research, the Air Force Research Laboratory (Rome, NY), Defense Advanced Research Projects Agency, and the Robert A. Welch Foundation (Grant No. A1261).
[1] S. E. Harris, J. E. Field, and A. Imamoglu, Phys. Rev. Lett. 64, 1107 (1990).

[2] M. O. Scully and M. S. Zubairy, Quantum Optics (Cambridge University Press, Cambridge, U.K., 1997).

[3] S. E. Harris and L. V. Hau, Phys. Rev. Lett. 82, 4611 (1999); S. E. Harris and Y. Yamamoto, ibid. 81, 3611 (1998); H. Schmidt and A. Imamoglu, Opt. Lett. 21, 1936 (1996).

[4] C. Liu, Z. Dutton, C. H. Behroozi, and L. V. Hau, Nature (London) 409, 490 (2001); D. F. Phillips, A. Fleischhauer, A. Mair, R. L. Walsworth, and M. D. Lukin, Phys. Rev. Lett. 86, 783 (2001); A. S. Zibrov, A. B. Matsko, O. Kocharovskaya, Y. V. Rostovtsev, G. R. Welch, and M. O. Scully, ibid. 88, 103601 (2002).

[5] M. S. Zubairy, A. B. Matsko, and M. O. Scully, Phys. Rev. A 64, 043804 (2001).

[6] M. Bahoura and A. Clairon, Opt. Lett. 26, 926 (2001).

[7] M. Martinelli, P. Valente, H. Failache, D. Felinto, L. S. Cruz, P. Nussenzveig, and A. Lezama, Phys. Rev. A 69, 043809 (2004)

[8] J. A. Armstrong, J. Opt. Soc. Am. 56, 1024 (1966).

[9] C. G. Alzar, L. Cruz, J. A. Gomez, M. F. Santos, and P. Nussenzveig, Europhys. Lett. 61, 485 (2003).
[10] M. D. Eisaman, A. Andre, F. Massou, A. S. Zibrov, and M. D. Lukin, Nature (London) 438, 837 (2005).

[11] V. A. Sautenkov, Y. V. Rostovtsev, and M. O. Scully, Phys. Rev. A 72, 065801 (2005).

[12] A. F. Huss, R. Lammegger, C. Neureiter, E. A. Korsunsky, and L. Windholz, Phys. Rev. Lett. 93, 223601 (2004).

[13] S. Sultana and M. S. Zubairy, Phys. Rev. A 49, 438 (1994).

[14] R. Coussement, Y. Rostovtsev, J. Odeurs, G. Neyens, H. Muramatsu, S. Gheysen, R. Callens, K. Vyvey, G. Kozyreff, P. Mandel, R. Shakhmuratov, and O. Kocharovskaya, Phys. Rev. Lett. 89, 107601 (2002).

[15] M. M. Kash, V. A. Sautenkov, A. S. Zibrov, L. Hollberg, G. R. Welch, M. D. Lukin, Yu. Rostovtsev, E. S. Fry, and M. O. Scully, Phys. Rev. Lett. 82, 5229 (1999).

[16] M. D. Lukin, M. Fleischhauer, A. S. Zibrov, H. G. Robinson, V. L. Velichansky, L. Hollberg, and M. O. Scully, Phys. Rev. Lett. 79, 2959 (1997).

[17] L. M. Duan, J. I. Cirac, P. Zoller, and E. S. Polzik, Phys. Rev. Lett. 85, 5643 (2000).

[18] R. B. Miles, A. P. Yalin, Z. Tang, S. H. Zaidi, and J. N. Forkey, Meas. Sci. Technol. 12, 442 (2001); N. D. Finkeistein, W. R. Lempert, and R. B. Miles, Opt. Lett. 22, 537 (1997). 